\title{
Blue metal-poor stars
}

\author{
George W. Preston ${ }^{1}$ and Christopher Sneden ${ }^{2}$ \\ ${ }^{1}$ Carnegie Observatories, 813 Santa Barbara Street, Pasadena, CA 91101, USA \\ email: gwp@ociw.edu \\ ${ }^{2}$ Department of Astronomy and McDonald Observatory, University of Texas, \\ Austin, TX 78712, USA \\ email: chris@verdi.as.utexas.edu
}

\begin{abstract}
We review the discovery of blue metal-poor (BMP) stars and the resolution of this population into blue stragglers and intermediate-age Main-Sequence stars by use of binary fractions. We show that the specific frequencies of blue stragglers in the halo field and in globular clusters differ by an order of magnitude. We attribute this difference to the different modes of production of these two populations. We report carbon and $s$-process enrichment among very metal-poor field blue stragglers and discuss how this result can be used to further resolve field blue stragglers into groups formed during RGB and AGB evolution of their erstwhile primary companions.
\end{abstract}

Keywords. Binaries: spectroscopic, blue stragglers, stars: chemically peculiar, stars: abundances

\section{Introduction: Identification of BMP stars}

In an isolated very old Milky Way Galactic halo there should be no Main Sequence stars of spectral type A-F. All these stars should have long-since evolved to become members of the white dwarf sequence. But hundreds of such objects that are collectively called blue metal-poor (hereafter BMP) stars have been discovered to date in Galactic halo photometric surveys, too many to be ignored in attempts to understand the origin and evolution of our Galaxy. These BMP stars comprise a laboratory for the investigations of stellar astrophysics in two very different environments: (1) in interacting halo binaries and (2) in (probably) dwarf satellite galaxies of the Milky Way. Numerous BMP stars were identified (Preston et al. 1994) among the metal-poor candidates and A-type stars of the HK survey (Preston et al. 1991) by their locations blueward of all globular cluster (hereafter GC) turnoffs in the $(U-B)$ vs $(B-V)$ plane, see Figure 1 of Preston et al. (1994). That paper employed a blue cutoff at $B-V=0.15$ because the $U-B$ blanketing effect provides little or no discrimination at bluer colors (higher temperatures). Hotter BMP stars may exist, but we do not know how to identify them in the halo field.

The BMP population possesses the photometric characteristics of the blue straggler (hereafter BS) families in globular clusters (Fusi Pecci et al. 1992), but it differs from them in important respects. The specific frequency of halo BMPs with $[\mathrm{Fe} / \mathrm{H}]<-1$, reckoned as the number of BMPs per horizontal branch star, exceeds the similarly defined specific frequency of GC BSs by a factor of 15 (Preston \& Sneden 2000, hereafter PS2000), so we know that these two samples of stars cannot have been drawn from the same parent population. Further understanding of the BMP phenomenon has come with spectroscopic investigations, and in this paper we review the results of two related high resolution studies by our group. 


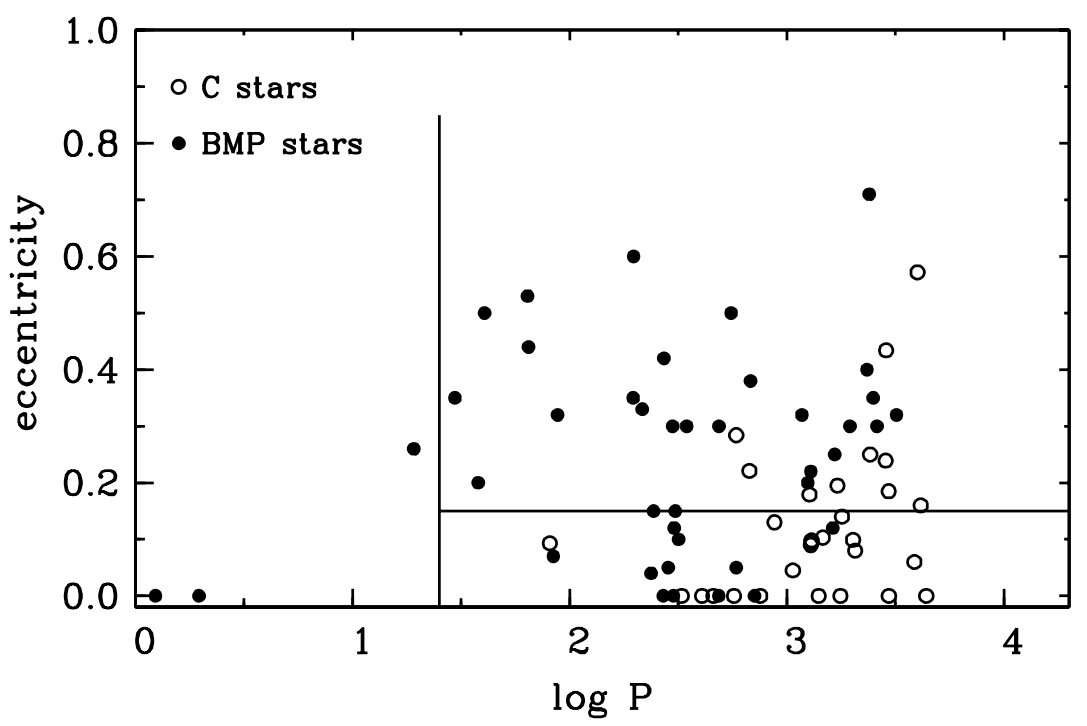

Figure 1. Correlation of binary orbital periods and eccentricities for BMP stars (PS2000), and in "C stars" (subgiant CH stars, McClure 1997; CH giants and barium stars, McClure \& Woodsworth 1990). This figure is an adaptation of the lower two panels of Figure 19 in PS2000. The vertical line drawn at $P=25$ days $(\log P=1.4)$ indicates the approximate transition period between short-period binaries that almost always have low-eccentricity orbits and long-period binaries that do not have strongly correlated periods and eccentricities. The horizontal line drawn at (an arbitrary) eccentricity $e=0.15$ represents an approximate lower limit to the eccentricities of binaries that have not undergone forced orbital circularization from their companions. For clarity in this figure, we have plotted only mean parameters for those few BMP stars with 2-3 alias periods listed by PS2000.

\section{RV-constant and binary BMP stars}

PS2000 conducted a radial velocity survey of 62 BMP stars, using high-resolution $R \equiv \lambda / \Delta \lambda \simeq 30000)$, low signal-to-noise $(S / N \sim 10)$ echelle spectra obtained with the Las Campanas du Pont Telescope. Between 10 and 70 individual spectra (on average $\simeq 20$ ) were gathered for each program star. Their velocity analysis revealed that $\simeq 60 \%$ of BMPs are in spectroscopic binaries with predominantly long periods. This binary frequency greatly exceeds the value of $15 \%$ found for spectroscopic binaries with orbital periods less than 4000 days in both the Galactic halo (Latham et al. 1998) and disk (Duquennoy \& Mayor 1991). Additionally, the fraction of double-lined spectroscopic binaries among BMPs is abnormally low (1 of 42 ), their mass functions are small, and an unusually large fraction of their orbits have low eccentricities. We illustrate these unexpected results in Figure 1, which compares the periods and eccentricities of BMPs to some known classes of carbon-rich binaries (McClure 1997, McClure \& Woodsworth 1990). Both groups of stars contain a large fraction of stars that have nearly circular orbits $(e<0.15)$ in spite of being in widely separated, long-period orbits $(P>25$ days). This distribution of eccentricities is very different from those of normal disk and halo binary stars (Duquennoy \& Mayor 1991, Latham et al. 1988, 1992), for which long-period, low-eccentricity orbits are rare.

Noting that the incidence of binaries among the BSs in M67 is very high, $>60 \%$ (Latham \& Milone 1996), and supposing that the BMPs are a mixture of BSs and a field population of intermediate-age stars with a normal (15\%) binary frequency, PS2000 calculated that $60 \%$ of the BMPs are field BSs, most of which were created by mass transfer during the evolution of their erstwhile primary companions (McCrea 1964). The 
specific frequency of the BS component of the BMPs exceeds the GC value by a factor of 10. We attribute this high value in the field to formation of BSs by mass transfer among long-period binaries which dominate the period distribution of field binaries (Duquennoy \& Mayor 1991). Formation of BSs by collisions and disruption of binaries by stellar encounters simply cannot occur in the very low-density halo. In GCs the specific frequency of BSs is low because preponderant wide binaries with long periods are destroyed by encounters in dense stellar systems: BS production is limited to the merger of the small population of short period survivors, an argument first made in a study of NGC 5466 by Mateo et al. (1990) and recently confirmed for 47 Tuc by Mapelli et al. (2004). A small percentage $(\simeq 10 \%)$ of primordial halo binaries with initial periods less than $\simeq 5$ days (Duquennoy \& Mayor 1991) merged (Vilhu 1982) to form more massive BSs, most or all of which left the Main Sequence long ago, so virtually all field blue stragglers are located in binaries, the products of mass transfer from erstwhile primaries that are now their degenerate companions. The binary BS population is contaminated by a small $(\simeq 10 \%)$ contribution due to the normal binaries that we presume accompany the intermediate-age field population.

We suggest that the single (RV-constant) BMP stars are the identifiable blue tips of intermediate-age populations captured from systems like the Carina dwarf galaxy (Smecker-Hane et al. 1994). Preston et al. (1994) estimated that such captured populations comprise perhaps $4-10 \%$ of the present halo. Subsequently, Unavane et al. (1996) drew a similar conclusion in a broader discussion of Galactic accretion.

\section{Chemical compositions of binary and RV-constant BMP stars}

Further insight into the two subclasses of BMP stars can be gained from their chemical compositions. PS2000 co-added the $\simeq 10-70$ individual echelle spectra of each of their BMP stars to produce moderate $S / N(\sim 30-40)$, adequate to derive $[\mathrm{Fe} / \mathrm{H}] \dagger$ metallicities and relative $[\mathrm{X} / \mathrm{Fe}]$ abundance ratios for: (a) Fe-peak elements $\mathrm{Sc}, \mathrm{Cr}$, and $\mathrm{Mn}$; (b) light $\alpha$-elements $\mathrm{Mg}, \mathrm{Ca}$, and $\mathrm{Ti}$; and (c) neutron-capture (n-capture) elements $\mathrm{Sr}$ and $\mathrm{Ba}$. Their observations included a sample of 62 stars, 19 of which rotate too fast $\left(V_{\mathrm{e}} \sin i>\right.$ $25 \mathrm{~km} \mathrm{~s}^{-1}$ ) to permit accurate determination of much more than their overall metallicity. The rapidly-rotating stars will not be considered further here. In Figure 2 we summarize the abundances for the remaining 43 stars, distinguishing the binaries from RV-constant stars via different symbols.

In the top panel of Figure 2 we show the mean abundance of the $\alpha$ elements $\mathrm{Mg}, \mathrm{Ca}$, and Ti for each star. For almost all stars, the "normal" Galactic halo-star enhancement of $[\alpha / \mathrm{Fe}] \simeq+0.3 \pm 0.1$ appears in BMP stars. $\neq$ No obvious separation is seen between the $\alpha$-element abundances of RV-constant and binary BMP stars. In the middle panel, the mean of Fe-peak elements Sc and Cr are displayed. The abundances of this element group in BMP stars are consistent with the values in other kinds of halo stars, and there is no difference between the two BMP subclasses. This statement extends also to Mn, which is not included in the Fe-peak means because it was not as well determined in the PS2000 study, with only unmeaningful upper limits recorded for many BMP stars.

The $n$-capture abundance means $<[\mathrm{Sr} / \mathrm{Fe}],[\mathrm{Bi} / \mathrm{Fe}]>$ shown in the bottom panel of Figure 2 are more interesting. At lowest metallicities, $[\mathrm{Fe} / \mathrm{H}]<-2$, the RV-constant BMP

$\dagger$ For elements A and B, standard abundance notation is used: $[\mathrm{A} / \mathrm{B}] \equiv \log _{10}\left(\mathrm{~N}_{\mathrm{A}} / \mathrm{N}_{\mathrm{B}}\right)_{\text {star }}-$ $\log _{10}\left(\mathrm{~N}_{\mathrm{A}} / \mathrm{N}_{\mathrm{B}}\right) \odot$

$\ddagger$ The one clear exception is the star CS 22966-043 $([\mathrm{Fe} / \mathrm{H}]=-1.96,[\alpha / \mathrm{Fe}]=-0.13$ in the top panel of Figure 2. This star is an example of the apparently rare subclass of $\alpha$-poor halo stars, discussed in detail by Ivans et al. (2003). 


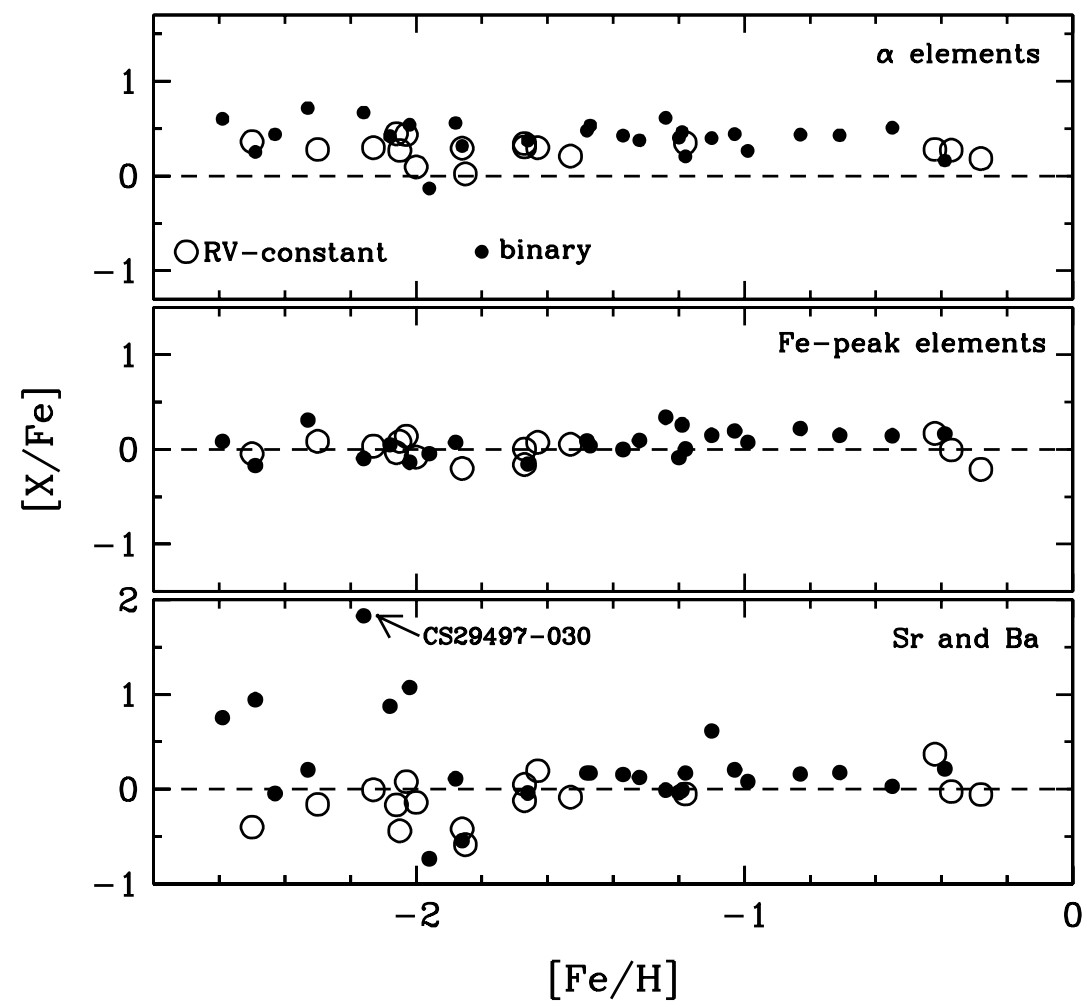

Figure 2. Mean abundances of different element groups in BMP stars derived from the survey data of PS2000. Abundances for their 43 slowly-to-moderately rotating stars $\left(V_{e} \sin i<\right.$ $25 \mathrm{~km} \mathrm{~s}^{-1}$ ) are plotted, but not for their 11 more rapidly rotating stars (for which abundances are less certain). Different symbols are used to distinguish BMP binary stars and those with constant radial velocities. In the upper panel the ordinate is the mean abundance of three $\alpha$ elements: $<[\mathrm{Mg} / \mathrm{Fe}],[\mathrm{Mg} / \mathrm{Fe}],[\mathrm{Ti} / \mathrm{Fe}]>$. In the middle panel the means are of two Fe-peak elements: $<[\mathrm{Sc} / \mathrm{Fe}],[\mathrm{Cr} / \mathrm{Fe}]>$. In the bottom panel the means are of two $n$-capture elements: $<[\mathrm{Sr} / \mathrm{Fe}],[\mathrm{Bi} / \mathrm{Fe}]>$. Attention is called to the star CS 29497-030 because it exhibits nearly a two-order-of-magnitude enhancement of these $n$-capture elements.

stars have no enhancements or perhaps deficiencies of the $n$-capture elements, while five out of the seven BMP binaries have very large enhancements of these elements. Sneden et al. (2003) obtained higher $S / N$ spectra for a sample of 10 BMP stars drawn from the PS2000 survey, five in each subclass. Of these, three binaries and three RV-constant BMP stars were very metal-poor, $[\mathrm{Fe} / \mathrm{H}] \sim-2.1$. Their study included the BMP binary CS 29497-030, which has the largest Sr and Ba abundance mean of the stars in Figure 2. The principle results of their abundance analysis can be summarized by inspection of the spectra displayed in Figure 3. In this figure we contrast the spectrum of CS 29497-030 to that resulting from the co-addition of the individual spectra of the three very metal-poor RV-constant stars. Although CS 29497-030 is the most extreme case, chosen deliberately for display purposes, almost all of the following conclusions apply to each of the three very metal-poor binaries.

(1) BMP binaries are carbon-rich. In Figure 3's top panel, we show some C I lines in CS 29497-030 that are absent in the RV-constant stars; the other two binaries also have detectable, albeit weaker $\mathrm{C}$ I lines. Additionally, the $\mathrm{CH}$ G-band can also be seen in all three binaries, but not in the RV-constant stars. In the BMP $\left(T_{\text {eff }}, \log g,[\mathrm{Fe} / \mathrm{H}]\right)$ regime, C-containing species should be completely undetectable. Noting that (high excitation) $\mathrm{C}$ 


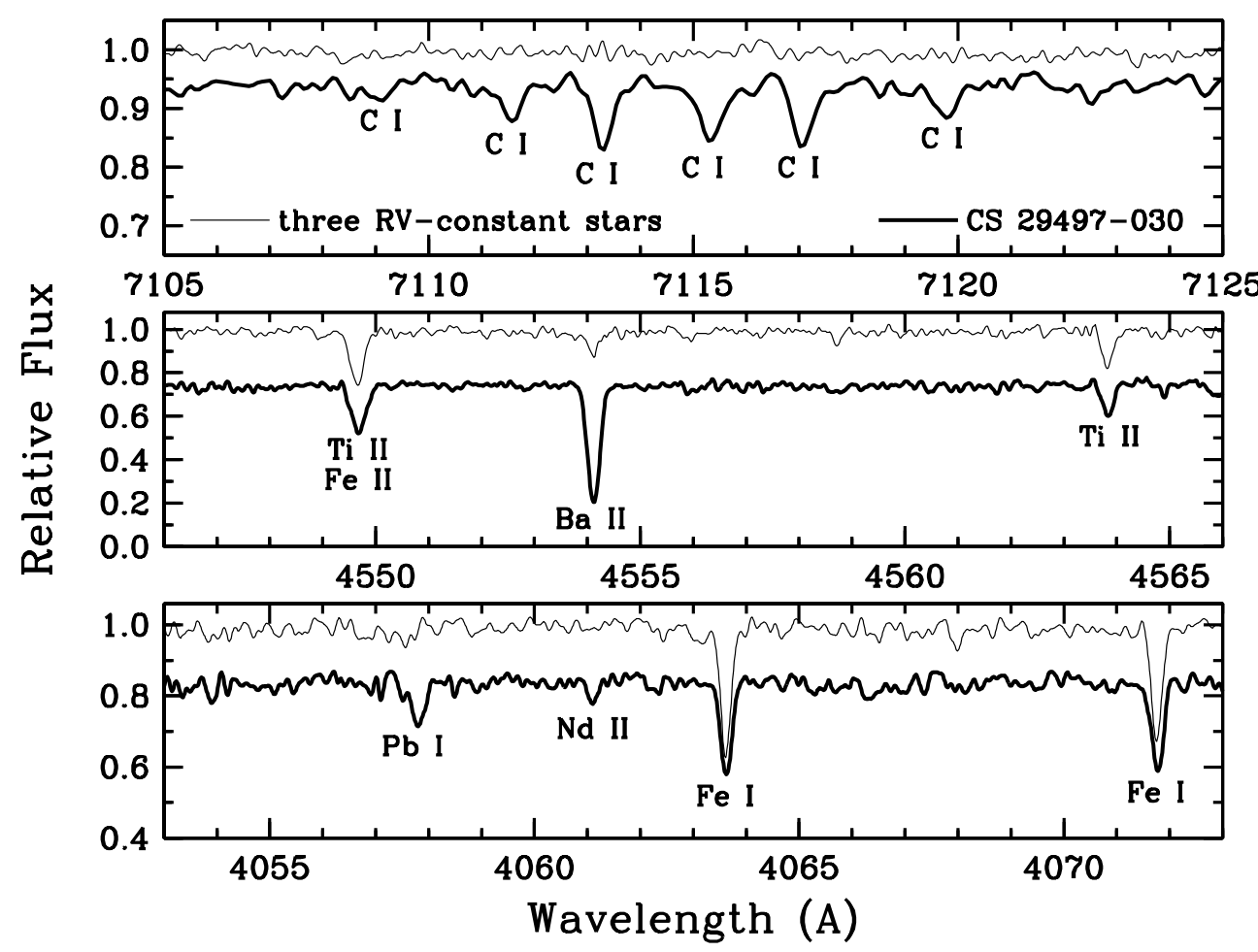

Figure 3. Spectra of selected small wavelength regions in the C-rich, $n$-capture-rich BMP binary star CS 29497-030, contrasted with co-added spectra of three RV-constant stars of comparable metallicity $([\mathrm{Fe} / \mathrm{H}] \sim-2)$. This figure is adapted from Figures 4 and 6 of Sneden et al. (2003). We emphasize that the mean RV-constant spectra have a complete absence of detectable $\mathrm{C}$ I lines (top panel), a very weak $\mathrm{Ba}$ II line (middle panel), and no sign of $\mathrm{Pb}$ I or $\mathrm{Nd}$ II (bottom panel), while those features are prominent in CS 29497-030 (and in the other two BMP binaries investigated by Sneden et al. 2003).

I naturally strengthens and $\mathrm{CH}$ weakens with increasing $T_{\text {eff }}$, clearly a pure abundance effect is at work. Carbon is enhanced by orders of magnitude in BMP binaries. (2) $B M P$ binaries are $n$-capture-rich. In the middle panel of Figure 3 we contrast the Ba II resonance line strength between CS 29497-030 and the RV-constant stars. While the PS2000 Ba abundance estimates were based on this single feature, the more detailed Sneden et al. (2003) investigation included 3-4 Ba II lines and other (3) Lead is present in BMP binary star CS 29497-030. In the bottom panel of Figure 3 we show the spectrum of the $\mathrm{Pb}$ I $\lambda 4057$ line; clearly this feature is strong (there are few stars with detectable $\mathrm{Pb} \mathrm{I}$ in their spectra). (4) The n-capture-element enhancements in BMP binaries are due to the s-process. Although Sr II and Ba II lines are extremely strong in the BMP binary spectra, lines of Eu II (not shown in Figure 3) are very weak or undetectable. Rapid $n$-capture nucleosynthesis (the $r$-process) produces Eu much more efficiently than $\mathrm{Ba}$, hence the lack of any Eu II detections immediately suggests that slow $n$-capture synthesis (the $s$-process) has been at work.

Sneden et al. (2003) determined abundances for nine $n$-capture elements in CS 29497$030([\mathrm{Fe} / \mathrm{H}]=-2.16)$, finding extremely large overabundances for most of these elements. For example, they derived $[\mathrm{Ba} / \mathrm{Fe}]=+2.45$ and $[\mathrm{Pb} / \mathrm{Fe}]=+3.75$, meaning that this very metal-poor star has a larger surface Ba content and more than a factor of 10 more $\mathrm{Pb}$ than does the Sun. Other Pb-rich stars have been discovered in recent years (e.g., 


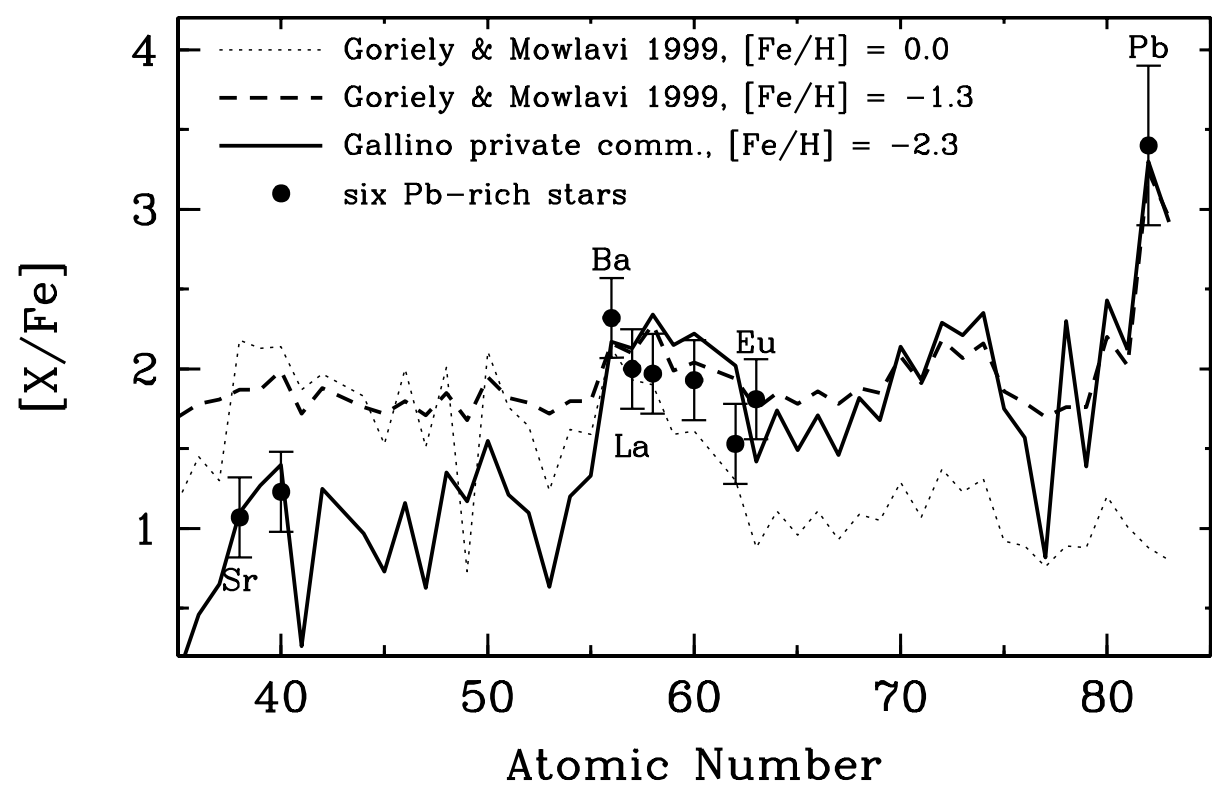

Figure 4. Reproduction of Figure 8 of Sneden et al. (2003): normalized mean $n$-capture abundances of six Pb-rich metal-poor stars (CS 22183-015, Johnson \& Bolte 2002; CS 29526-110, Aoki et al. 2002; HD 187861 and HD 196944, Van Eck et al. 2003; HE 0024-2523, Lucatello et al. 2003; CS 29497-030, Sneden et al. 2003), and theoretical s-process predictions for nucleosynthesis environments of three different metallicities (Goriely \& Mowlavi 2000, Gallino 2003). See the discussion in Sneden et al. (2003) for a detailed description on formation of the mean observed abundances.

Johnson \& Bolte 2002, Aoki et al. 2002, Van Eck et al. 2003, Lucatello et al. 2003), these other stars are most often evolved red giants. The Pb-rich stars have various $[\mathrm{Ba} / \mathrm{Pb}]$ ratios, but the general $n$-capture abundance patterns are very similar in all of them. In Figure 4 we show Figure 8 of Sneden et al. (2003) that compares six of the stars with largest $\mathrm{Pb}$ abundances to several $s$-process synthesis predictions. One of the $s$-process theoretical curves was computed by Goriely \& Mowlavi (2000) for a solar-metallicity fusion environment, one was produced by these same authors for a moderately metalpoor $([\mathrm{Fe} / \mathrm{H}]=-1.3)$ environment, and one is an unpublished prediction calculated by $\mathrm{R}$. Gallino (private communication) for a very metal-poor $([\mathrm{Fe} / \mathrm{H}]=-1.3)$ regime. Clearly the best match is with the Gallino $s$-process predications, because these were made with the lowest assumed metallicity (comparable to that of CS 29497-030). The explanation, originally due to Clayton (1988) and developed quantitatively by, e.g., Travaglio et al. (1999, and references therein), suggests that as the metallicity decreases in an $s$-process synthesis zone, the ratio of free neutrons to available seed nuclei increases. This naturally drives the $s$-process neutrons to attach to fewer target nuclei, pushing the resultant abundance distribution to heavier nuclei. For sufficiently low metallicities, an $s$-process piles up at $\mathrm{Pb}$ (and $\mathrm{Bi}$ ), the heaviest stable nuclei in the Periodic Table.

\section{Putting it all together}

Statistical inferences from the HK survey, and recent radial velocity and abundance investigations all confirm that field BMP stars comprise two distinct halo subpopulations. The capture origin of the RV-constant stars remains as the only viable explanation for their presence in the halo, because we cannot imagine how these metal-poor stars could have been produced recently within our Galaxy. 
The origin of the BMP BS binary component seems more clear. All evidence points such BMP stars being the end product of post Main Sequence mass transfer. The stars seen today originally possessed less than or about the mass of an ordinary 12-15 Gyr metal-poor halo turnoff star. Their companions were probably much more massive, up to perhaps several solar masses (we cannot be more precise on the basis of present spectroscopic data, though some abundance details do depend on the mass of the primordial primary that undergoes helium shell burning). As the erstwhile primaries evolved, their outer envelopes expanded until they overflowed their Roche lobes and transferred mass to their companions, which in response moved to a brighter and bluer portion of the Main Sequence; the original primaries are now white dwarfs. Transfer could occur during RGB or AGB evolution. The existence of $\mathrm{C}$ and $s$-process abundance enrichments in some (most?) of the most metal-poor BMP binaries guarantees that often this event occurred during the AGB "third dredge-up" evolutionary phase. The BMPs with long orbital periods present challenges for theoretical interpretation. Systems with periods greater than about 300 days or more should complete RGB evolution without Roche-lobe overflow. Therefore, we expect that the preponderance of the stars with periods greater than some cutoff value (not precise because of the unknown initial primary mass) should show AGB peculiarities to some degree. The expectations are clouded because AGB superwinds offer a second path to mass transfer. The amount of mass that can be transferred by winds in widely separated pairs is small, but then the contaminated convective layers of the present rather warm BMP primaries are shallow. The future of this topic rests on better theoretical calculations that connect plausible mass transfer to better abundance estimates based on superior spectra.

On the purely observational side, orbital parameters are not in a satisfactory state. The orbital periods derived in PS2000 are plagued by alias problems associated with annual trips to Las Campanas Observatory. Furthermore, a number of stars in our sample exhibit low-amplitude velocity variations on time scales comparable to and in some cases exceeding the time duration of our survey. Therefore a proper discussion of mass transfer in such wide systems must await additional velocity data that will lead to definitive orbits.

The wherewithal for improved BMP spectroscopy is now greatly enhanced with the completion of a number of new, large Southern Hemisphere telescope facilities (Gemini, Magellan, SOAR, VLT). We hope that some time at these facilities will be devoted to the issues raised in this presentation.

\section{Acknowledgements}

Some of the studies reviewed here occurred while C. S. was a Visiting Scientist at the Carnegie Observatories; their hospitality and financial support are gratefully acknowledged. This research has been supported in part by NSF grants AST-9987162 and AST-0307495 to C. S.

\section{References}

Aoki, W., Ryan, S.G., Norris, J.E., Beers, T.C., Ando, H., \& Tsangarides, S. 2002, ApJ 580, 1149

Clayton, D.D. 1988, MNRAS 234, 1

Duquennoy, A., \& Mayor, M. 1991, A\&A 248, 485

Fusi Pecci, F., Ferraro, F.R., Corsi, C.E., Cacciari, C., \& Buonanno, R. 1992, AJ 104, 1831

Gallino, R. 2003, private communication

Goriely, S. \& Mowlavi, N. 2000, A $\& A$ 362, 599 
Ivans, I.I., Sneden, C., James, C.R., Preston, G.W., Fulbright, J.P., Höflich, P.A., Carney, B.W., \& Wheeler, J.C. 2003, ApJ 592, 906

Johnson, J.A. \& Bolte, M.B. 2002, ApJ 579, L87

Latham, D.W., \& Milone, A.E. 1996, ASP Conf. Ser. 90, The Origins, Evolution, and Destinies of Binary Stars in Clusters, ed. E. F. Milone \& J.-C. Mermilliod (San Francisco: Astr. Soc. Pac.), 385

Latham, D.W., Mazeh, T., Carney, B.W., McCrosky, R.E., Stefanik, R.P., \& Davis, R.J. 1988, AJ 96,567

Latham, D. W., et al. 1992, AJ 104, 774

Latham, D.W., Stefanik, R.P., Mazeh, T., Goldberg, D., Torres, G., \& Carney, B.W. 1998, ASP Conf. Ser., 154, Cool Stars, Stellar Systems, and the Sun, ed. R. A. Donahue \& J. A. Bookbinder (San Francisco: Astr. Soc. Pac.) 2129

Lucatello, S., Gratton, R., Cohen, J.G., Beers, T.C., Christlieb, N., Carretta, E., \& Ramírez, S. 2003, $A J 125,875$

Mapelli, M., Sigurdsson, S., Colpi, M., Ferraro, F.R., Possenti, A., Rood, R.T., Sills, A., \& Beccari, G. 2004, ApJ 605, L29

Mateo, M., Harris, H.C., Nemec, J. \& Olszewski, E.W. 1990, AJ 100, 469

McClure, R.D. 1997, PASP 109, 536

McClure, R.D. \& Woodsworth, A.W. 1990, ApJ 352, 709

McCrea, W.H. 1964, MNRAS 128, 147

Preston, G.W., Beers, T.C., \& Shectman, S.A. 1994, AJ 108, 538

Preston, G.W., Shectman, S.A., \& Beers, T.C. 1991, ApJ.S 76, 1001

Preston, G.W. \& Sneden, C. 2000, AJ 120, 1014

Smecker-Hane, T.A., Stetson, P.B., Hesser, J.E., \& Lehnert, M.D. 1994, AJ 108, 507

Sneden, C., Preston, G.W., \& Cowan, J.J. 2003, ApJ 592, 504

Travaglio, C., Galli, D., Gallino, R., Busso, M., Ferrini, F., \& Straniero, O. 1999, ApJ 521, 691

Unavane, M., Wyse, R.F.G., \& Gilmore, G. 1996, MNRAS 278, 727

Van Eck, S., Goriely, S., Jorissen, A., \& Plez, B. 2001, Nature 412, 793

Vilhu, O. 1982, A\&\&A 109, 17

\section{Discussion}

LANDSTREeT: Your abundance plots of $[\mathrm{X} / \mathrm{H}]$ vs. $[\mathrm{Fe} / \mathrm{H}]$ show a fairly uniform distribution of stars down to $[\mathrm{Fe} / \mathrm{H}] \approx-2.6$ where the sample ends abruptly. Why does the sample stop there, rather than continuing to lower metallicities?

PRESTON: Stars of lower metallicity are simply hard to find. There is no other reason for the lower bound to our Fe abundances.

WeIss: Can you please comment on the errors of the individual factors you were using in estimating the BMP incidence and how errors would propagate?

PRESTON: I would estimate that the errors in the specific frequencies of field and globular cluster blue stragglers are both typically 10 to 20 per cent - far smaller than the orderof-magnitude difference between the frequencies of the two groups.

MÖHLER: Why do you compare the blue straggler frequency among the BMP stars to the BSS frequency in globular clusters? Wouldn't open clusters be more appropriate, considering the dynamical conditions?

PRESTON: The abundances, spatial distributuions and kinematical characteristics of the BMP stars have a classical halo signature. This is why we use the globular clusters as reference systems. The BS's in M 67, however, appear to have the binary frequencies that we have adopted for our BS population. 